\title{
Effects of Obesity on Posaconazole Kinetics in a Patient with Graft versus Host Disease
}

\author{
Justin J. Pettis ${ }^{1}$, Stephanie R. Hyche ${ }^{1}$, Katherine N. Logan ${ }^{1}$, Arash Poursina ${ }^{2}$, P. Brandon Bookstaver ${ }^{{ }^{*}}$ \\ ${ }^{1}$ South Carolina College of Pharmacy, University of South Carolina, Columbia, USA; ${ }^{2}$ Aurora Sheboygan Memorial Medical Center, \\ Sheboygan, USA. \\ Email: *bookstaver@sccp.sc.edu
}

Received January $18^{\text {th }}, 2013$; revised February $25^{\text {th }}, 2013$; accepted April $4^{\text {th }}, 2013$

Copyright (c) 2013 Justin J. Pettis et al. This is an open access article distributed under the Creative Commons Attribution License, which permits unrestricted use, distribution, and reproduction in any medium, provided the original work is properly cited.

\begin{abstract}
Posaconazole, a triazole antifungal agent, displays a highly variable pharmacokinetic profile due in part to the oral solution, the only currently available dosage form. Posaconazole activity appears to be dose-dependent and clinical outcomes correlate with steady-state serum trough concentrations and area under the concentration curve (AUC) over minimum inhibitory concentration (MIC) ratios. Therapeutic drug monitoring (TDM) has been recommended to help overcome the unpredictable nature of the drug. The impact of obesity of posaconazole pharmacokinetics and the use of TDM in this population has not been well described; however, limited data demonstrate increased weight correlates with lower serum concentrations and reduced drug exposure. We describe the use of TDM in an obese man, 12 months status post hematopoietic stem-cell transplant (HSCT), receiving posaconazole therapy for a presumed invasive fungal infection (IFI).
\end{abstract}

Keywords: Posaconazole; Obesity; Pharmacokinetics; Therapeutic Drug Monitoring

\section{Introduction}

Over the last 20 years, obesity in the United States has increased at an alarming rate. Greater than one-third of American adults (35.7\%) and 12.5 million children and adolescents aged 2 to 19 years old are classified as obese [1]. Appropriate drug therapy in the obese population can present a difficult challenge for clinicians, as many drugs, including antimicrobials, require an altered dosing modality due to significant changes in pharmacokinetic parameters [2].

Posaconazole, a triazole antifungal agent structurally and chemically similar to itraconazole, displays a highly variable pharmacokinetic profile due in part to the oral solution, the only currently available dosage form. Apparent volume of distribution ranges from 5 to $25 \mathrm{~L} / \mathrm{kg}$ suggesting extensive tissue distribution [3,4]. Posaconazole activity appears to be dose-dependent and clinical outcomes correlate with steady-state serum trough concentrations and area under the concentration curve (AUC) over minimum inhibitory concentration (MIC) ratios $[3,4]$. Enhanced drug absorption up to 4-fold in the presence of food, specifically a high fat meal, has been well

\footnotetext{
"Corresponding author.
}

described [3,4]. Conversely, serum concentrations are reduced in patients receiving concomitant acid reducing agents and in the presence of concurrent states such as diarrhea and mucositis [4]. Therapeutic drug monitoring (TDM) has been recommended to help overcome the unpredictable nature of the drug $[5,6]$.

The impact of obesity of posaconazole pharmacokinetics and the use of TDM in this population has not been well described; however, limited data demonstrate increased weight correlates with lower serum concentrations and reduced drug exposure $[7,8]$. We describe the use of TDM in an obese man, 12-month status post hematopoietic stem-cell transplant (HSCT), receiving posaconazole therapy for a presumed invasive fungal infection (IFI).

\section{Case Report}

We present the case of a 52-year-old obese Caucasian man admitted for mouth ulcers and fever persisting three to four weeks. His past medical history was significant for mantle cell lymphoma, diabetes mellitus, chronic kidney disease and obesity (weight $109 \mathrm{~kg}$ and body mass index $35.6 \mathrm{~kg} / \mathrm{m}^{2}$ ). The patient was diagnosed with 
stage IV-B mantel cell lymphoma in 2001. Following multiple relapses the patient underwent an autologous stem cell transplant (SCT) in 2010, followed by an allogeneic SCT in 2011 due to persistent disease. At that time, he was initiated on prophylactic voriconazole 200 mg PO BID, valacyclovir 2 grams PO daily, atovaquone $1500 \mathrm{mg}$ PO daily and tacrolimus $0.5 \mathrm{mg}$ PO daily. The patient presented with complaints of mouth ulcers and odynophagia resulting in multiple emergency room visits and hospitalizations over the course of four weeks. Swabs of the ulcers were negative for CMV, HSV $1 / 2$ and Varicella. Buccal tissue and adjacent molar cultures were positive for light growth of Candida glabrata. The patient received courses of oral acyclovir, oral amoxicillin/clavulanate and nystatin mouthwash without resolution.

On the admission of interest, vital signs were as follows: temperature of $100.7^{\circ} \mathrm{F}$; respiratory rate, 20 per minute; heart rate, 100 per minute; blood pressure 143/80 mmHg. Baseline laboratory workup revealed a WBC count of $3.9 \mathrm{~K} / \mathrm{uL}$ with $74 \%$ neutrophils, $5 \%$ lymphocytes, $5 \%$ metamyelocytes and $16 \%$ monocytes. A chest computed tomography scan on hospital day (HD) 1 revealed centrally located alveolar infiltrates of the upper and lower lungs. The ulcers were biopsied and the pathology was consistent with Graft-versus-host disease (GVHD), believed to be complicated by a presumed opportunistic pneumonia. Swabs of the ulcers were again negative for HSV 1/2 and CMV. Bronchoalveolar lavage cultures revealed only C. glabrata; blood cultures, and all acid fast bacilli respiratory cultures were negative. Susceptibility testing was not conducted as the C. glabrata was not presumed to be pathogenic. Prophylactic valacyclovir, atovaquone and tacrolimus were continued and posaconazole $400 \mathrm{mg}$ PO twice daily was initiated on HD 2. Additional concomitant medications included amlodipine, carvedilol, colchicine, esomeprazole, fenofibrate, insulin aspart and glargine, magnesium oxide, meropenem and vancomycin. During his hospitalization, liver and renal function remained normal. The patient tolerated a soft mechanical diet throughout and intake remained consistent with meals scheduled in concert with posaconazole doses.

Due to the patient's obesity, concomitant use of esomeprazole and the unpredictable pharmacokinetic profile of the formulation, TDM (performed at Fungal Testing Laboratory, University of Texas Health Center, San Antonio, TX) was obtained on HD 6 (following 9 doses) which revealed posaconazole concentrations of $0.6 \mathrm{mcg} / \mathrm{mL}, 0.6$ $\mathrm{mcg} / \mathrm{mL}$ and $0.8 \mathrm{mcg} / \mathrm{mL}$ at 1.4 hours prior to 0800 dose, 1 hour prior to 1700 dose, and 3.8 hours after 1700 dose, respectively (Figure 1). Results of the TDM were not available until HD 13. Posaconazole therapy was discontinued on HD 9 at which time the patient's symptoms were determined to be secondary to GVHD rather than an opportunistic fungal infection. Despite attempted therapy, progressive GVHD led to the patient's death approximately 2 months later.

\section{Poseconazole Serum Concentrations}

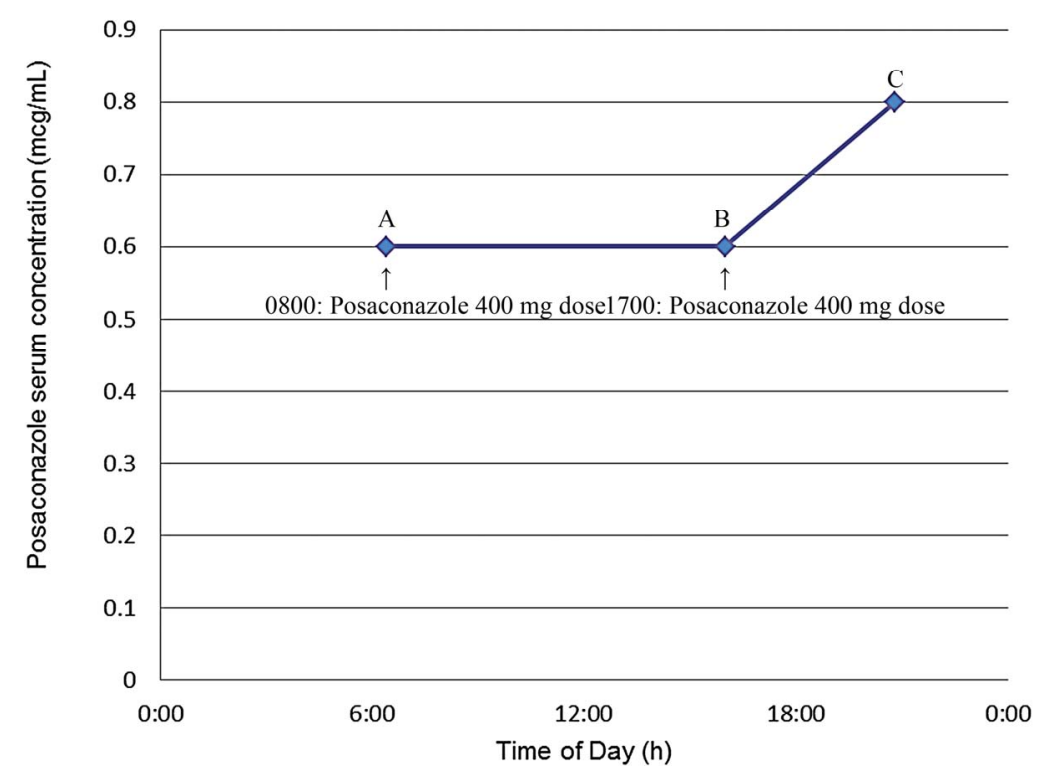

${ }^{a}$ Therapeutic drug monitoring performed at Fungal Testing Laboratory, University of Texas Health Center, San Antonio, TX. A = $0.6 \mathrm{mcg} / \mathrm{mL}$ obtained 1.4 hours prior to $0800 \mathrm{dose} ; \mathrm{B}=0.6 \mathrm{mcg} / \mathrm{mL}$ obtained 1 hour prior to 1700 dose; $\mathrm{C}=0.8 \mathrm{mcg} / \mathrm{mL}$ obtained 3.8 hours post 1700 dose.

Figure 1. Posaconazole therapeutic drug monitoring in case patient following multiple doses ${ }^{\mathrm{a}}$. 


\section{Discussion}

Obesity is associated with physiological alterations that grossly affect drug pharmacokinetics [2]. Drug distribution may be affected due to an increased volume of blood and cardiac output, cardiac alterations, and an increased volume of distribution [2,9]. Depending on the extent of obesity, changes in protein binding can be altered, renal clearance may be increased or decreased and changes in hepatic metabolism may affect the elimination of the agent. Posaconazole is $98 \%$ - $99 \%$ protein bound to primarily albumin [3]. In obese patients, the lipophilicity of posaconazole may contribute to its distribution into excess adipose tissue, thereby decreasing circulating free drug concentrations [3,4]. Additionally, obese patients have increased renal clearance, which when coupled with a total-body-weight-proportional increase in glucuronidation and sulfation, may also lead to decreased exposure to posaconazole [2].

Serum concentrations in our patient were significantly lower than steady-state concentrations obtained on day 14 of therapy in healthy volunteers, but similar to those reported in an obese renal transplant patient receiving $200 \mathrm{mg}$ four times daily [7,8]. Our obese case patient was maintained on a consistent diet throughout hospitalization, but, of note, was receiving proton pump inhibitor therapy which has been shown to decrease posaconazole concentrations by approximately $30 \%$ [4]. Target serum concentrations have been suggested at 0.7 $\mathrm{mcg} / \mathrm{mL}$ and $1.25 \mathrm{mcg} / \mathrm{mL}$, respectively, for prophylaxis and treatment of invasive Aspergillus infections [10,11]. AUC/MIC ratios of approximately 400 and potentially up to 1000 may be required to optimize posaconazole activity against Aspergillus species [3,12,13]. Based on these targets, our patient would likely have had suboptimal concentrations in the treatment of an IFI, which may have compromised desired outcomes.

Clinical outcomes have been correlated with higher posaconazole serum concentrations $[6,11,14]$. In a population of cardiothoracic transplant (CTT) patients, Shields et al. demonstrated significantly higher median trough concentrations, $1.55 \mathrm{mcg} / \mathrm{mL}$ vs. $0.34 \mathrm{mcg} / \mathrm{mL}$ ( $\mathrm{p}=$ 0.006), in patients with a positive therapeutic outcome compared to those with treatment failure, respectively [14]. Dolton et al. demonstrated similar outcomes in SCT patients receiving posaconazole for prophylaxis or treatment. In the subset of patients receiving posaconazole for IFI, patients with successful outcomes had higher median serum concentrations compared to those with poor treatment outcomes, $0.955 \mathrm{mcg} / \mathrm{mL}$ vs. $0.436 \mathrm{mcg} / \mathrm{mL}$, respectively [6]. Reasons for suboptimal concentrations most commonly include drug-drug interactions, such as acid-suppressing or prokinetic agents. Weight has also been associated with decreased posaconazole exposure in prospective evaluations. In a PK study of SCT patients with GVHD, Krishna et al. demonstrated a $28 \%$ reduction $(1.128 \mathrm{mcg} / \mathrm{mL}$ vs. $0.814 \mathrm{mcg} / \mathrm{mL})$ in median serum concentrations between patients $<65 \mathrm{~kg}$ compared to those $>80 \mathrm{~kg}$ [15]. Additionally, in the study by Dolton and colleagues mentioned above, overweight CTT patients had lower mean serum concentrations compared to other patients, $0.86 \mathrm{mcg} / \mathrm{mL}$ vs. $0.46 \mathrm{mcg} / \mathrm{mL}, \mathrm{p}=0.04$ [6]. For the reasons mentioned previously, obesity may compromise the ability to achieve desired serum concentrations.

Dose modification in obese patients may be considered, however saturation has been shown to occur at doses above $800 \mathrm{mg}$ daily [3,4]. Posaconazole in divided doses given $200 \mathrm{mg}$ four times daily provides higher AUC compared to $400 \mathrm{mg}$ administered twice daily, as used in our case, and may be an option in obese patients $[3,4]$. However, despite an even further increase to $200 \mathrm{mg}$ six times daily, Kuipers et al. showed no additional increase in serum trough concentrations $(0.6 \mathrm{mcg} / \mathrm{mL})$ in an obese renal transplant recipient [8]. In a small subset of patients, Shields et al. demonstrated an increase in serum concentrations when total daily doses were increased to 1600 mg daily [14]. Although not used extensively, therapeutic drug monitoring has been suggested for many patients receiving posaconazole therapy, although obesity was not specifically encouraged as a potential justification for TDM [5].

Variations in pharmacokinetics are well known with posaconazole oral suspension. The presence of obesity may increase the risk of suboptimal drug exposure, compromising desired clinical outcomes. Maximizing physiologic conditions and minimizing drug-drug interactions should be widely employed in individuals on posaconazole therapy, despite similarities or differences to our case patient. Clinicians should be aware that modifying posaconazole regimens, including dosage increases, may not produce the target serum concentrations in obese patients. We support the use of therapeutic drug monitoring for obese patients, especially those with concomitant factors associated with reduced posaconazole exposure. It is not known if our specific case is representative of other obese subjects receiving posaconazole. Further study is necessary in order to determine the optimal posaconazole dosing regimen and correlation with outcomes in obese patients. Use of the new tablet formulation, currently under evaluation, may help to alleviate some of the expected variation in pharmacokinetics; however, confirmation in obese patients will still be required [16].

\section{Acknowledgements}

We acknowledge Dr. Sharon Weissman's contribution to patient management. 


\section{Transparency Declarations}

PBB: Research funding from Cubist Pharmaceuticals ${ }^{\circledR}$ (antimicrobial lock therapy); Research funding from Merck \& Co., Inc. (anti-fungal lock therapy); Research funding from Astellas US Pharma ${ }^{\circledR}$ (antifungal lock therapy); Advisory Board for Optimer Pharmaceuticals ${ }^{\circledR}$ (fidaxomicin); Speaker's bureau for Merck \& Co., Inc. (non-branded, antibiotic stewardship) JP, SH, KL, AP: Nothing to disclose.

No funding was received related to this patient case or manuscript production. Actions were carried out in the normal course of patient care.

\section{REFERENCES}

[1] CDC Centers for Disease Control and Prevention, "Overweight and Obesity," 2012. http://www.cdc.gov/obesity/data/facts.html

[2] M. J. Hanley, D. R. Abernethy and D. J. Greenblatt, “Effect of Obesity on the Pharmacokinetics of Drugs in Humans," Clinical Pharmacokinetics, Vol. 49, No. 2, 2010, pp. 71-87. doi:10.2165/11318100-000000000-00000

[3] D. Andes, K. Marchillo, R. Conklin, et al., "Pharmacodynamics of a New Triazole, Posaconazole, in a Murine Model of Disseminated Candidiasis," Antimicrobial Agents and Chemotherapy, Vol. 48, No. 1, 2004, pp. 137-142. doi:10.1128/AAC.48.1.137-142.2004

[4] Y. Li, U. Theuretzbacher, C. J. Clancy, M. H. Nguyen and H. Derendorf, "Pharmacokinetic/Pharmacodynamic Profile of Posaconazole,” Clinical Pharmacokinetics, Vol. 49, No. 6, 2010, pp. 379-396. doi:10.2165/11319340-000000000-00000

[5] S. J. Howard, T. W. Felton, A. Gomez-Lopez and W. W. Hope, "Posaconazole: The Case for Therapeutic Drug Monitoring," Therapeutic Drug Monitoring, Vol. 34, No. 1, 2012, pp. 72-76. doi:10.1097/FTD.0b013e31823cdeac

[6] M. J. Dolton, J. E. Ray, D. Marriott and A. J. McLachlan, "Posaconazole Exposure-Response Relationship: Evaluating the Utility of Therapeutic Drug Monitoring," Antimicrobial Agents and Chemotherapy, Vol. 56, No. 6, 2012, pp. 2806-2813. doi:10.1128/AAC.05900-11

[7] R. Courtney, S. Pai, M. Laughlin, J. Lim and V. Batra, "Pharmacokinetics, Safety, and Tolerability of Oral Posaconazole Administered in Single and Multiple Doses in Healthy Adults," Antimicrobial Agents and Chemotherapy, Vol. 47, No. 9, 2003, pp. 2788-2795. doi:10.1128/AAC.47.9.2788-2795.2003

[8] S. Kuipers, R. J. M. Brüggemann, R. G. L. de Sévaux, et al., "Failure of Posaconazole Therapy in a Renal Trans- plant Patient with Invasive Aspergillosis Due to Aspergillus fumigatus with Attenuated Susceptibility to Posaconazole,” Antimicrobial Agents and Chemotherapy, Vol. 55, No. 7, 2011, pp. 3564-3566. doi:10.1128/AAC.01544-10

[9] R. Wurtz, G. Itokazu and K. Rodvold, “Antimicrobial Dosing in Obese Patients,” Clinical Infectious Diseases, Vol. 25, No. 1, 1997, pp. 112-118. doi:10.1086/514505

[10] T. J. Walsh, I. Raad, T. J. Patterson, et al., “Treatment of Invasive Aspergillosis with Posaconazole in Patients who Are Refractory to or Intolerant of Conventional Therapy: An Externally Controlled Trial," Clinical Infectious Diseases, Vol. 44, No. 1, 2007, pp. 2-12. doi:10.1086/508774

[11] S. H. Jang, P. M. Colangelo and J. V. Gobburu, "Exposure-Response of Posaconazole Used for Prophylaxis against Invasive Fungal Infections: Evaluating the Need to Adjust Doses Based on Drug Concentrations in Plasma," Clinical Pharmacology \& Therapeutics, Vol. 88, 2010, pp. 115-119. doi:10.1038/clpt.2010.64

[12] E. Mavridou, R. J. Bruggemann, W. J. Melchers, J. W. Mouton and P. E. Verweij, "Efficacy of Posaconazole against Three Clinical Aspergillus fumigatus Isolates with Mutations in the CYP51A Gene," Antimicrobial Agents and Chemotherapy, Vol. 54, No. 2, 2010, pp. 860-865. doi:10.1128/AAC.00931-09

[13] S. J. Howard, J. M. Lestner, A. Sharp, L. Gregson, J. Goodwin, J. Slater, J. B. Majithiya, P. A. Warn and W. W. Hope, "Pharmacokinetics and Pharmacodynamics of Posaconazole for Invasive Pulmonary Aspergillosis: Clinical Implications for Antifungal Therapy,” The Journal of Infectious Diseases, Vol. 203, No. 9, 2011, pp. 1324-1332. doi:10.1093/infdis/jir023

[14] R. K. Shields, C. J. Clancy, A. Vadnerkar, et al., "Posaconazole Serum Concentrations among Cardiothoracic Transplant Recipients: Factors Impacting trough Levels and Correlation with Clinical Response to Therapy," Antimicrobial Agents and Chemotherapy, Vol. 55, No. 3, 2011, pp. 1308-1311. doi:10.1128/AAC.01325-10

[15] G. Krishna, M. Martinho, P. Chandrasekar, A. J. Ullmann and H. Patino, "Pharmacokinetics of Oral Posaconazole in Allogeneic hematopoietic Stem Cell Transplant Recipients with Graft-versus-Host Disease," Pharmacotherapy, Vol. 27,No. 12, 2007, pp. 1627-1636.

[16] G. Krishna, L. Ma, M. Martinho, R. A. Preston and E. O’Mara, “A New Solid Oral Tablet Formulation of Posaconazole: A Randomized Clinical Trial to Investigate Rising Single- and Multiple-Dose Pharmacokinetics and Safety in Healthy Volunteers," Journal of Antimicrobial Chemotherapy, Vol. 67, No. 11, 2012, pp. 2725-2730. doi:10.1093/jac/dks268 